\title{
Biochemical Definition of the Uremic Syndrome and Possible Therapeutic Implications
}

\author{
R. Vanholder, ${ }^{*}$ C. Hsu, and S. Ringoir \\ Nephrology Department, University Hospital, Ghent, Belgium, and ${ }^{*}$ Nephrology Division, University of Michigan \\ Medical School, Ann Arbor, Michigan, U.S.A.
}

Renal failure is characterized by the progressive decline in the capacity of the kidneys to eliminate toxic solutes. In parallel, the "uremic syndrome" is depicted by a functional disturbance of enzymes, organelles, cells, and organs. This leads to an overall malaise that ultimately ends in coma and death unless artificial kidney treatment (dialysis) is started (1).

The current conception of dialysis is highly aspecific. Emphasis has been on the elimination of small, water-soluble compounds such as creatinine and urea; however, the toxicity of the latter substances is still debatable. Uremic biological fluids are in fact complex mixtures containing innumerable interfering compounds that are the basis of the "uremic syndrome." The uremic toxin probably does not exist (2).

Despite several decades of study, the pathophysiology of the uremic syndrome remains ill defined. The possibilities in this domain have been improved recently by the introduction of new techniques for biochemical and biological evaluation and for separation and identification of the contents of complex biological fluids. The latter steps were made possible by the introduction of high-performance liquid chromatography (HPLC). In our laboratory, a stepwise strategy has been developed whereby complex uremic mixtures (sera or ultrafiltrates) are fractionated in several fractions (eluates). By evaluating the effect of uremic biological fluids on functional systems that are known to be disturbed in renal failure, we can pursue a fractionation of these biological fluids to identify the responsible compounds. This approach has already enabled us to identify the sub-

Received November 1992.

Address correspondence and reprint requests to Dr. R. Vanholder at the Nephrology Department, University Hospital, De Pintelaan 185, B9000 Ghent, Belgium. stances responsible for changes in the functional capacity of biochemical systems with important clinical implications in end-stage renal failure: metabolization of calcitriol (relevant in treatment of renal bone disease); phagocytic polymorph metabolic function (relevant in susceptibility to infection); and drug-protein binding (relevant in the efficacy and toxicity of drug treatment).

This enabled us to better define the pathophysiology of these important clinical problems, which remained partly or entirely unresolved up to now. Subsequent efforts can be focused on a better definition and on improvement of the treatment of uremia by dialysis.

Identification of the factors responsible for renal failure and improvement of the uremic condition have important clinical as well as socioeconomic implications (more than 1,000,000 patients are treated by renal replacement therapy worldwide).

\section{THE INFLUENCE OF UREMIC ULTRAFILTRATE ON CALCITRIOL METABOLISM}

Decreased production of calcitriol in renal failure is believed to be a major factor in the development of hyperparathyroidism, hypocalcemia, and renal osteodystrophy characteristic of chronic kidney disease. Although loss of renal tissue is a primary cause of decreased calcitriol production in renal failure by a decrease in available $1 \alpha$-hydroxylase, it was demonstrated that uremic plasma ultrafiltrate also suppresses the production of calcitriol in normal rats (3). Uremic substances, not further defined, were also considered to play a major role in the changes in the metabolic clearance rate of calcitriol (3). Thus, uremic biological fluids contain factors that affect production and degradation of calcitriol.

Our studies in this domain had as their main pur- 
pose the attempt to define the factor(s) responsible for these defects.

\section{Uremic ultrafiltrate factors affect the metabolization of calcitriol}

To characterize the substances responsible for the suppression of the production and metabolic clearance of calcitriol in normal rats, uremic plasma ultrafiltrates were fractionated using HPLC (gradient of formic acid [pH 4.0$] 100 \%$ to methanol $80 \%$ in 52 $\mathrm{min}$ ) to study the effect of each fraction on calcitriol metabolism (3). Parameters of calcitriol metabolism were measured after an infusion of these fractions and of radiolabeled calcitriol to normal rats. The HPLC-elution patterns of the fractions with a suppressive effect suggest that at least two groups with distinguishable chemical characteristics are responsible: Fraction 4, containing small water-soluble compounds (mainly purines), and Fractions 6 to 13, containing mainly lipophilic, partially protein-bound substances. The molecular weight of the substances under evaluation, in view of the optimum separation expected for the HPLC technique used, is below 2,000 daltons. One of the 13 fractions, Fraction 4, contains uric acid, xanthine, and hypoxanthine. Infusion of subfractions of Fraction 4 markedly re duced both the production and the metabolization of calcitriol.

We conclude from this study that both the production and the degradation of calcitriol are influenced by solutes retained in uremic sera and ultrafiltrate. The previously held belief that such metabolic changes, especially in production of calcitriol, are due solely to a decrease in renal mass is contradicted. At least two groups of substances are responsible. One of these may be related to the purines; the other group is less well defined but is characterized by an elution time in the second half of the HPLC system used, which suggests larger molecular weight, partial lipophilicity and/or hydrophobicity, and/or substantial protein binding.

\section{Purine derivatives influence calcitriol metabolization in rats}

The following study checks the relation suggested above between retention of purines in renal failure and suppression of metabolic production and clearance rate of calcitriol (4). Therefore, calcitriol metabolism was evaluated with or without the infusion of purines to healthy rats (4). The calcitriol production rate and clearance were significantly reduced after infusion of either theophylline $(1 \mathrm{mg} / \mathrm{h})$ or sodium urate $(0.5 \mathrm{mg} / \mathrm{h})$ compared with those in control rats infused with normal saline. Renal $1 \alpha$-hy- droxygenase activity of kidney homogenate was significantly inhibited in rats infused with theophylline and urate. Administration of the purines uric acid and theophylline causes a decrease in plasma calcitriol concentration in view of the more important decrease in production than in degradation.

We conclude from this study that purine derivatives suppress calcitriol synthesis. These data thus confirm the "toxic" interference of the purines with calcitriol metabolization, especially for uric acid, which in general and despite repeated study has always been considered as a nontoxic uremic solute.

Uric acid levels inversely related to plasma calcitriol in patients with chronic renal failure

In view of the finding of a decrease of calcitriol levels with increasing uric acid concentrations in normal rats, the effect of a decrease of uric acid levels of patients in end-stage renal failure was studied (5). After 1 week of treatment with allopurinol, $300 \mathrm{mg}$ daily, plasma uric acid decreased significantly. The plasma concentration of calcitriol, however, rose in each patient. Allopurinol per se had no effect on calcitriol metabolism.

Administration of allopurinol suppresses plasma uric acid and increases plasma calcitriol in patients with chronic renal failure. Thus, uremia and hyperuricemia could reduce the biological action of calcitriol on subsequent metabolic processes in patients with renal failure, and the implications of these findings may go beyond the scope of the mere effect of uric acid on calcitriol metabolism alone (e.g., effect of calcitriol on parathormone synthesis and on bone metabolism). Therefore, the use of allopurinol in the prevention of renal osteodystrophy in chronic renal failure should be further investigated.

\section{PHAGOCYTE METABOLIC ACTIVITY}

Infection is a frequent complication and a major cause of death among patients with end-stage renal failure. Polymorphonuclear phagocytes are important in host defense mainly because of bacterial destruction by NADPH-oxidase-related free radical production following phagocytosis. Studies were undertaken to evaluate the leukocyte response to stimuli by an evaluation of the glycolytic activity, in which the $\mathrm{CO}_{2}$ production by the hexose monophosphate shunt (HMS) in the energy delivery to NAD (P) $\mathrm{H}$-oxidase is measured, and by estimations of production of free radical species.

\section{Prospective cross-sectional study in a population with renal failure}

The leukocyte response to phagocytic challenge was assessed in uremic and hemodialysis patients 
in a prospective cross-sectional study (6). Using latex and zymosan as phagocytic challenge, the utilization of glucose $-1-{ }^{14} \mathrm{C}$ and the generation of reactive oxygen species were measured in these patients. In uremic, non-dialysis-dependent patients, the response to phagocytosis was significantly reduced when serum creatinine exceeded $6 \mathrm{mg} / \mathrm{dl}$, and prior to initiation of dialysis, it was less than half that of patients with normal renal function. In maintenance dialysis patients, despite enhanced elimination of uremic solutes, a similar suppression is observed. This decreased response to phagocytic stimulus could be used as a predictor of hospitalization for infectious reasons, especially overt bacteremias, as patients developing major infection necessitating hospitalization had significantly lower response. In a prospective study of 15 patients initiated on dial$y$ sis, the metabolic response of their leukocytes was assessed sequentially, and complement-activating Cuprophan membranes were compared with noncomplement-activating polysulfone. The choice of the Cuprophan membrane was inspired by the finding that this dialysis membrane induces activation of complement factors: the question that arises then is whether this activation leaves the polymorphs with the ability to cope with further "natural stimuli" once this prestimulation has taken place.

In 8 patients, initiation of dialysis with the complement-activating Cuprophan membrane led to a further decline $(60 \%)$ in metabolic response to phagocytosis at the end of 4 weeks of dialysis compared with that before initiation of dialysis; however, in 7 other patients on dialysis with non-complement-activating membranes, this decline was largely attenuated. Prospective crossover studies with fluorescence analysis in response to dichlorofluorescein-diacetate (a molecule that becomes fluorescent upon contact with oxygen-reactive species) and flow cytometry corroborated these findings.

We conclude that there is in renal failure a profound disturbance in polymorph metabolic activity in response to phagocytosis. This disturbance is multifactorial and is related to the progressive development of the uremic status as well as to the start of hemodialysis treatment. In relation to hemodialysis, the progressive functional decline is more profound in patients treated with complement-activating $\mathrm{Cu}$ prophan than in those treated with non-complement-activating polysulfone. These changes are correlated to the hospitalization rate for major infection. The risk of host-defense disturbances in the phagocytic immune system and the subsequent risk of infection thus increase with advancing uremia and with the use of the Cuprophan membrane.

\section{Acute effects of hemodialysis on}

phagocytic response

In a second study, an in vivo and in vitro evaluation was undertaken comparing healthy control subjects and dialyzed renal failure patients and evaluating the polymorph metabolic response to five different stimuli with different pathways for activation of polymorphonuclear function: latex, zymosan, Staphylococcus aureus, phorbol myristic acid, and f-Met-Leu-Phen (7). Our results show a marked and parallel inhibition in the response to these five stimuli in predialysis samples. This response is further suppressed after $15 \mathrm{~min}$ of Cuprophan dialysis for all stimuli $(-40$ to $-94 \%)$ except phorbol myristic acid. Polymorphonuclear response remains intact during dialysis with non-complement-activating dialyzers. In vitro experiments confirm decreased polymorphonuclear glycolytic activity after the suspension of Cuprophan fragments in normal whole blood.

This study confirms the impairment of polymorphonuclear cell energy delivery to NADPH-oxidase in patients with end-stage renal failure. The impaired response to various stimuli is different in predialysis blood samples and samples collected during Cuprophan dialysis, so the inhibition observed in these two different conditions may be related to different mechanisms.

\section{Effect of uremic biological fluids on polymorph function}

In view of the progressive decline of polymorph $\mathrm{NAD}(\mathrm{P}) \mathrm{H}$-related glycolysis during advancing uremia, it was evaluated whether this effect could be attributed to a compound (or compounds) retained in uremic biological fluids. Therefore, isolated normal and uremic polymorphs were suspended in normal and uremic plasma in a matched crossover study evaluating polymorph glycolytic activity in response to latex, zymosan, and $S$. aureus. An optimal response was obtained when normal cells were suspended in normal plasma whereas the response was minimal when uremic cells were suspended in uremic plasma (8). The responses of normal cells in uremic plasma and of uremic cells in normal plasma were intermediate. These data indicate that uremic plasma suppresses polymorph metabolism. Uremic ultrafiltrate caused a similar suppression.

\section{Effect of recombinant erythropoietin on polymorph metabolic capacity}

Erythropoietin has recently been introduced for the treatment of uremic anemia. In theory, only effects on the erythroid cell line can be expected. To 
evaluate the influence of erythropoietin on granulocyte function, a double-blind, randomized, placebocontrolled study was undertaken in 22 patients on maintenance hemodialysis (9). A marked and progressive improvement was observed after a mean treatment period of 4 months. A significant correlation between hematocrit and ${ }^{14} \mathrm{CO}_{2}$ production was observed in the erythropoietin-treated group. No such correlation was found with serum ferritin.

The depressed phagocyte glycolytic activity of hemodialyzed uremic patients is normalized during correction of anemia by erythropoietin. This may be attributed to a better metabolic condition. Since in the present study no major changes in serum ferritin were obtained and the improvement was not correlated to serum ferritin but only to hematocrit, reduction of body iron stores may be a less important contributing factor.

\section{DRUG-PROTEIN BINDING}

A better understanding of the factors influencing the protein binding of drugs during renal failure has implications in the field of efficacy and toxicity of drug treatment as only free drugs can be considered to be pharmacologically active. In addition, drugprotein binding in renal failure is possibly decreased by not fully defined organic acid compounds. These organic acids interfering with drug-protein binding reportedly will alter the accumulation of other organic acids in specific organs (e.g., the brain) as well as in the entire body; in addition, they widen the "anion gap," which further adds to their toxic impact. Thus, inhibition of protein binding and failure of organic anion transport may be related to each other.

\section{Screening evaluation of patients with chronic renal failure}

In a first study, changes of the protein-binding properties of 9 drugs were evaluated by an ultrafiltration technique (Centrifree) that indicates the fraction of free versus total radioactive drug in plasma solution after the ultrafiltration maneuver (10). In addition, theophylline and phenytoin, two drugs with the most substantial and progressive decrease in protein binding, were further studied by HPLC fractions of ultrafiltrate of normal and uremic serum in an attempt to identify substances causing inhibition of drug-protein binding. Ultrafiltrate obtained during a hemofiltration session inhibited protein binding of theophylline and phenytoin in a dosedependent way. After separation of this ultrafiltrate by HPLC, it appeared that for both theophylline and phenytoin at least a part of this inhibitory activity corresponded to the elution zone of hippuric acid. For theophylline, two other inhibitory zones were further recognized: one corresponding to the elution zone of $\mathrm{NaCl}$ and one in which the responsible substance remained unidentified. Hippuric acid in solution inhibited protein binding of theophylline and phenytoin in a dose-dependent way.

In conclusion, protein binding of several drugs currently used in the treatment of renal failure is affected in parallel with renal function, which might alter the effectiveness of the drugs. Hippuric acid appears to play an important role in the proteinbinding defect of theophylline and phenytoin.

\section{Comparative studies of deproteinization methods}

Studies of factors affecting drug-protein binding based on ultrafiltrate have the disadvantage that by definition the heaviest protein binders are omitted from analysis. In addition, in our former analysis, HPLC fractionation was arrested at $30 \mathrm{~min}$ (i.e., at a 50:50 formate: methanol gradient) whereas the bulk of protein-bound substrate can be expected to be eluted more to the right on these chromatograms (i.e., for an eluate composition with more than $50 \%$ methanol). We therefore decided to repeat the same study as described above but with deproteinized uremic sera and with more extended chromatograms. We found, however, no reliable published data on the ligand yield of different currently used deproteinization methods. Therefore, ligand extraction achieved after 12 different deproteinization methods was evaluated by comparison of ultraviolet-absorbing peaks on high-performance liquid chromatograms of uremic serum after deproteinization or ultrafiltration (11). In a first comparison, the number of peaks recovered and the number of significant peaks increasing by at least $25 \%$ in height after deproteinization were markedly higher with the heat denaturation, acetonitrile extraction, trichloroacetic acid, bilirubin displacement, and acid precipitation/ acetonitrile extraction methods. A more detailed comparison of the effects of these 5 methods on the calculated protein binding of 14 peaks showed that the yield differed from method to method and from peak to peak and that the highest ligand extraction or yield resulted from the heat denaturation method.

In conclusion, the yield of deproteinized ligand and the estimated protein binding of ligands differs with the deproteinization method used. More attention should be paid to the specific deproteinization methods used in studies of uremic ligand extraction. The use of more than one deproteinization method 
for studies of drug and ligand protein binding in uremia should enhance the reliability of the results.

\section{Influence of deproteinized serum samples on theophylline-protein binding}

Starting from this experience, we evaluated whether deproteinized uremic serum yielded more important inhibition of theophylline's ${ }^{14} \mathrm{C}$ protein binding compared with uremic ultrafiltrate. Two deproteinization methods were evaluated: heat denaturation and acetonitrile extraction (12). The addition of 0.1 to $1.5 \mathrm{ml}$ of lyophilized deproteinized uremic serum resulted in a more important decrease in theophylline-protein binding than did uremic ultrafiltrate.

In conclusion, theophylline-protein binding is suppressed more importantly by deproteinized uremic serum than by uremic ultrafiltrate.

\section{IMPLICATIONS IN THE DOMAIN OF HEMODIALYSIS}

Validity of urea and other markers of dialysis efficiency

To appreciate the validity of azotemic markers as an index for intradialytic solute concentration changes, 8 solutes (pseudo-uridine, PS; xanthine, XA; hypoxanthine, HX; peak 4, P4; peak 5, P5; p-OH hippuric acid, $\mathrm{PH}$; indoxyl sulfate, IS; and hippuric acid, HA) were compared to 5 classic azotemic markers (urea, UR; creatinine, CR; uric acid, $\mathrm{UA}$; phosphate, $\mathrm{P}$; and potassium, K) (13). Concentration was determined by reversed-phase HPLC coupled to UV adsorption or by photometric analysis. Regression analysis revealed a significant intermutual correlation $\left(p<10^{-5}\right)$ for a cluster of 7 compounds including urea as a classic parameter of dialysis adequacy as well as PS, UA, P4, P5, PH, and CR. The behavior of hippuric acid (HA) was specific in that its changes in concentration before and after dialysis correlated with this cluster of 7 but also with IS, HX, and K, as well as with the overall group of nonidentified UV-absorbing HPLC peaks accumulating in uremia. Solute concentration changes were different in two differing blood purification strategies, namely hemodialysis and hemodiafiltration, for hippuric acid and $\mathrm{p}-\mathrm{OH}$ hippuric acid but not for urea and creatinine.

We conclude that urea is only partially representative as a marker of the dialytic concentration changes of other retention compounds and that alternative markers such as hippurate, measured either by colorimetry or by HPLC peak height analysis (14), should be considered. This is especially im- portant in view of our findings of the interference of several solutes with biological systems, many of which have a different intradialytic behavior than urea. Therefore, an optimum solute elimination should be pursued, not only of the water-soluble, urea-like compounds but also of the partially lipophilic, larger compounds-mainly organic acids.

These data further suggest that it is of value to introduce a marker of uremic solute retention in addition to urea to account for renal-function-related, "organic-acid-like" compounds that are eliminated by renal tubular excretion in dialyzed patients. The hippurates may serve this purpose.

\section{CONCLUSION}

These data shed new light on the understanding and treatment of the uremic syndrome. Uremic intoxication is a complex, multifactorial problem. Several different metabolic systems are affected. The disturbances in metabolic functions are reflected in clinical problems (e.g., phagocytosis vs. infection risk, metabolization of calcitriol vs. osteogenesis, drug-protein binding vs. therapeutic efficacy). The responsible solutes depend on the metabolic system involved. Many of the solutes affecting metabolism have retention and elimination characteristics that differ from those of current markers like urea and creatinine. Classic approaches to the evaluation of toxin retention and removal are in part inaccurate. Fractionation of uremic biological fluids by HPLC methods adds new technological possibilities for the better definition of the uremic syndrome. Accurate prevention of morbidity and mortality can only be pursued when this multifactorial etiologic nature of the uremic syndrome is taken into account. Our data encourage pursuit of better elimination of organic acid and lipophilic compounds and of the purines, which are groups of compounds that are currently disregarded in the definition of uremia. In addition, it should be stressed that dialysis treatment, although by definition life-saving, may exert its own toxicity, which can be avoided by the use of specific "biocompatible" membrane types. Treatment with drugs (allopurinol, erythropoietin) is another alternative to cope with some of these problems.

The consideration of these factors should improve the survival and quality of life of the patient with end-stage renal failure.

\section{REFERENCES}

1. Vanholder RC, Ringoir S. The uraemic syndrome. In: Cameron S, Davison AM, Grünfeld JP, Kerr D, Ritz E, eds. 
Oxford textbook of clinical nephrology. Oxford, UK: Oxford Medical Publications, 1992:1236-50.

2. Vanholder R, Ringoir S. Adequacy of dialysis, a critical analysis. Kidney Int 1992;42:540-58.

3. Hsu CH, Vanholder R, Patel S, De Smet R, Sandra P, Ringoir SMG. Subfractions in uremic plasma ultrafiltrate inhibit calcitriol metabolism. Kidney Int 1991;40:868-73.

4. Hsu CH, Patel SR, Young EW, Vanholder R. Effects of purine derivatives on calcitriol metabolism in rats. $A m J$ Physiol 1991;260:F596-601.

5. Vanholder R, Hsu CH. Effect of uric acid on plasma levels of calcitriol in chronic renal failure [Abstract]. J Am Soc Nephrol 1991;2:246.

6. Vanholder R, Ringoir S, Dhondt A, Hakim R. Phagocytosis in uremic and hemodialysis patients: A prospective and cross sectional study. Kidney Int 1991;39:320-7.

7. Vanholder R, Dell'Aquila R, Jacobs V, Dhondt A, Veys N, Waterloos MA, Van Landschoot N, Van Biesen W, Ringoir S. Depressed phagocytosis in hemodialyzed patients: In vivo and in vitro mechanisms. Nephron (in press).

8. Vanholder R, Dell'Aquila R, Waterloos MA, De Smet R, Van Landschoot N, Ringoir S. Inhibition of phagocytosis by uraemic plasma and ultrafiltrate [Abstract]. Nephrol Dial Transplant 1991;6:788-9.

9. Veys N, Vanholder R, Ringoir S. Correction of deficient phagocytosis during erythropoietin treatment in maintenance hemodialysis patients. Am $J$ Kidney Dis $1992 ; 19: 358-63$.

10. Vanholder R, Van Landschoot N, De Smet R, Schoots A, Ringoir $\mathrm{S}$. Drug protein binding in chronic renal failure: Evaluation of nine drugs. Kidney Int 1988;33:996-1004.

11. Vanholder R, Höffliger N, De Smet R, Ringoir S. Extraction of protein bound ligands from azotemic sera: Comparison of 12 deproteinization methods. Kidney Int 1992;41:1707-12.

12. De Smet R,Vanholder R, Ringoir S. Theophylline protein binding inhibition by uraemic toxins [Abstract]. Nephrol Dial Transplant 1991;6:786-7.

13. Vanholder RC, De Smet RV, Ringoir SM. Validity of urea and other "uremic markers" for dialysis quantification. Clin Chem 1992;38:1429-36.

14. Vanholder R, De Smet R, Schoots A, Ringoir S. Correlation of a colorimetric method and a HPLC method for the determination of serum hippuric acid concentrations in uremia. Nephron 1988;49:164-8. 